\title{
25 septembre: I'avenir de la Suisse en jeu
}

Les médecins n'ont aucune raison de se faire du souci, nos concitoyennes et concitoyens non plus. La campagne en vue de la votation du 25 septembre se concentre sur des aspects purement spéculatifs qui tiennent d'une vision par le petit bout de la lorgnette. L'immigration médicale existe déjà dans notre pays avant et après les accords de libre circulation avec l'UE des Quinze. L'apport de collègues étrangers dans les hôpitaux (25\% environ en moyenne, davantage dans les hôpitaux universitaires) est indispensable à leur fonctionnement. La question de l'installation est différente, régulée qu'elle est par la clause du besoin pendant encore trois ans. Une déferlante polonoslovaque ou balte n'est donc pas pour demain.

La pratique médicale en cabinet est étroitement liée à une compréhension socio-culturelle dont la langue n'est que l'un des éléments. Les expériences accumulées dans l’UE jusqu'à présent démontrent que les mouvements migratoires médicaux sont extrêmement ténus. Ils se limitent à des appels d'offres en bonne et due forme pour compenser des pénuries préoccupantes comme au nord de l'Ecosse et de la Norvège et quelques spécialités très demandées (oncologie, chirurgie, anesthésie, etc.). Pour le reste, il s'agit de phénomènes limités comme entre l'Espagne et le Portugal qui offre de meilleures conditions de travail. L'essentiel concerne surtout des collègues retraités qui s'installent sur les côtes méditerranéennes, italiennes, françaises ou espagnoles et prennent soins de quelques compatriotes. Mais cela n'a aucune incidence significative sur les systèmes de sécurité sociale eux-mêmes.

Les concitoyennes et concitoyens qui craignent de perdre leur emploi au gré d'un dumping salarial mettent la charrue devant les bœufs. Les mesures d'accompagnement interdisent ce genre de situation, les conventions collectives négociées par les syndicats s'appliquant à tous y compris aux candidats européens. Il en va de même pour les assistants et chefs de clinique.

Par contre le refus de l'extension de la libre circulation des personnes aux dix pays nouveaux membres de l'UE aurait des effets désastreux. Ceux qui craignaient de perdre leur emploi risquent alors effectivement de se retrouver au chômage en raison de la crise économique certaine que déclencherait cette décision. Quoi qu'on en dise, chaque accord bilatéral, transports terrestres, transports aériens, marchés publics, agriculture, normes techniques, libre circulation des personnes et la recherche, est muni de la même clause guillotine: le refus d'un seul d'entre eux entraîne la dénonciation en cascade de tous les autres. Le Traité de Rome précise dans son article 12 «est interdite toute discrimination exercée en raison de la nationalité». L'idée que la Suisse puisse conclure des accords séparés avec une partie de l'UE seulement et pas avec le reste est totalement farfelue, voire xénophobe.

Les tenants du non prétendent que l'UE n'osera pas ... Cela relève de l'irresponsabilité la plus totale, pour ne pas utiliser d'autres qualitatifs moins respectueux. En cas de non, l'UE engagera d'abord la Suisse à la réflexion et examinera de concert avec le Conseil fédéral quelle solution pourrait être trouvée. Revotation ou dénonciation? Ou alors, et très probablement, il sera décidé d'un commun accord de donner du temps au temps. Les accords en vigueur ne seront peut-être pas dénoncés. Cela renverrait à la case de départ et obligerait à une nouvelle négociation complète. Il est fort douteux que nous parvenions aux résultats déjà obtenus dans ces conditions. Il est plus probable que les accords soient simplement gelés. Mais les effets seront les mêmes: la Suisse sera totalement isolée, ce qui aurait des effets désastreux au niveau de la coopération scientifique, de l'agriculture, de la poursuite de la construction des tunnels ferroviaires alpins, sans parler du financement de nos assurances sociales, AVS/AI en particulier, qui souffriront directement de la chute de la croissance.

Même atténué par quelques mesures dont on ne voit pas très bien ce qu'elles pourraient être, ce scénario resterait une catastrophe pour la Suisse. Repliée sur elle-même derrière des frontières verrouillées, ses échanges et son développement vont se heurter à des barrières politicoadministratives peut-être encore franchissables, mais suffisantes pour perdre parts de marché et crédit politique. Il n'y a pas de progrès possible sans coopération dans tous les domaines. Les accords bilatéraux ne sont pas une panacée, puisqu'ils ne peuvent entrer en vigueur qu'avec l'approbation individuelle de chaque état membre de l'UE et pas seulement celle des Suisses. Mais ils garantissent pour le moment l'acquis et n'hypothèquent aucune autre solution ou perspective future. Il est important que les médecins suisses se mobilisent. Il y va de l'avenir de notre pays, de notre profession et de la recherche scientifique. Je les engage vivement à voter OUI le 25 septembre prochain.

Dr Yves Guisan, vice-président FMH, conseiller national 


\section{September: Die Zukunft der Schweiz steht auf dem Spiel}

Ärztinnen und Ärzte haben ebensowenig Anlass zur Sorge wie unsere Mitbürgerinnen und Mitbürger: Die Kampagne für die Abstimmung vom 25. September konzentriert sich auf reine Spekulationen, die von einem Scheuklappendenken geprägt sind. Eine Ärztezuwanderung gab es bereits vor und nach dem Inkrafttreten des Abkommens über die Personenfreizügigkeit mit der 15 Mitglieder zählenden EU. Die Mitarbeit ausländischer Kollegen in den Spitälern - durchschnittlich rund $25 \%$, in den Universitätsspitälern sogar noch mehr ist für deren reibungslosen Betrieb unverzichtbar. Die Niederlassung in der eigenen Praxis ist ein anderes Thema, weil diese während dreier Jahre noch durch den Zulassungsstopp geregelt ist. Es wird also morgen nicht zu einer Einwanderungswelle von Ärzten aus Polen, der Slowakei oder den baltischen Staaten kommen.

Die Arbeit in der Arztpraxis ist eng mit einem soziokulturellen Verständnis verbunden. Die Sprache ist dabei nur ein Bestandteil. Die in der EU bisher gesammelten Erfahrungen zeigen, dass die Migrationsbewegungen bei den Ärzten äusserst gering sind. Sie beschränken sich auf ordnungsgemäss durchgeführte Ausschreibungen zur Behebung eines besorgniserregenden Ärztemangels (wie beispielsweise im Norden Schottlands und Norwegens) sowie auf einige sehr gefragte Fachrichtungen wie Onkologie, Chirurgie oder Anästhesie. Ansonsten handelt es sich um sehr begrenzte Erscheinungen wie z.B. zwischen Spanien und Portugal, das bessere Arbeitsbedingungen bietet. Im wesentlichen betrifft die Migration vor allem Kollegen im Ruhestand, die sich an der Mittelmeerküste in Italien, Frankreich oder Spanien niederlassen und einige Landsleute betreuen. Dies hat aber keinerlei bedeutsame Auswirkung auf die Systeme der sozialen Sicherheit.

Die Mitbürgerinnen und Mitbürger, die wegen Lohndumpings um ihren Arbeitsplatz fürchten, spannen den Pflug vor den Ochsen. Die flankierenden Massnahmen verhindern dies, weil die von den Gewerkschaften ausgehandelten Gesamtarbeitsverträge auf alle, auch auf die europäischen Kandidaten, anwendbar sind. Dies gilt ebenfalls für die Assistenz- und Oberärzte.

Würde hingegen die Ausdehnung der Personenfreizügigkeit auf die zehn neuen EU-Mitglieder abgelehnt, hätte dies katastrophale Folgen. Diejenigen, die um ihren Arbeitsplatz fürchten, könnten dann wegen der Wirtschaftskrise, die ein solcher Entscheid sicherlich auslösen würde, tatsächlich arbeitslos werden. Was auch immer behauptet wird: Jedes bilaterale Abkommen - Landverkehr, Luftverkehr, öffentliche Ausschreibungen, Landwirtschaft, technische Normen, Personenfreizügigkeit und Forschung - enthält die gleiche Guillotineklausel:
Die Ablehnung eines einzigen Abkommens führt zur Kündigung aller anderen. Gemäss Artikel 12 des Vertrags von Rom ist «jede Diskriminierung aus Gründen der Staatsangehörigkeit verboten». Die Vorstellung, die Schweiz könnte mit nur einem Teil der EU und nicht mit allen Mitgliedern getrennte Verträge abschliessen, ist völlig absurd, wenn nicht gar fremdenfeindlich.

Die Gegner behaupten, die EU würde es nicht wagen. Ein solches Argument zeugt von vollständiger Verantwortungslosigkeit, und dies ist noch milde ausgedrückt. Bei einem Nein würde die EU die Schweiz zum Nachdenken auffordern und zusammen mit dem Bundesrat überlegen, welche Lösung gefunden werden kann. Neue Abstimmung oder Kündigung? Sehr wahrscheinlich würde man gemeinsam beschliessen, die Sache erst einmal ruhen zu lassen. Die geltenden Verträge würden vielleicht nicht gekündigt. Damit stünden wir aber wieder am Ausgangspunkt und wären gezwungen, neue, vollständige Verhandlungen zu führen. Dabei ist höchst zweifelhaft, ob wir unter solchen Umständen die gleichen Ergebnisse erzielen würden. Wahrscheinlicher ist, dass die Abkommen einfach eingefroren würden. Die Wirkung wäre dieselbe: Die Schweiz wäre vollständig isoliert, was katastrophale Folgen für die wissenschaftliche Zusammenarbeit, die Landwirtschaft und den Weiterbau der Alpenbahntunnels hätte - ganz zu schweigen von der Finanzierung unserer Sozialversicherungen, insbesondere der AHV und IV, die direkt unter dem Wachstumsrückgang leiden würden.

Selbst mit einigen Massnahmen, die man sich eigentlich gar nicht richtig vorstellen kann, bliebe dieses Szenario eine Katastrophe für die Schweiz. Abgeschottet hinter geschlossenen Grenzen stünden der Handel und die Entwicklung des Landes vor politischen und administrativen Hindernissen, die vielleicht zu überwinden, aber genügend hoch wären, dass die Schweiz Marktanteile und politische Glaubwürdigkeit verlöre. Ohne Zusammenarbeit auf allen Gebieten ist kein Fortschritt möglich. Die bilateralen Abkommen sind kein Allheilmittel, weil sie nur in Kraft treten können, wenn ihnen ausser der Schweiz jedes Mitglied der EU einzeln zustimmt. Sie garantieren jedoch für den Augenblick das Erreichte und erschweren weder eine andere Lösung noch belasten sie die Zukunftsaussichten. Es ist wichtig, dass sich die Schweizer Ärztinnen und Ärzte mobilisieren. Es geht um die Zukunft unseres Landes, unseres Berufes und der wissenschaftlichen Forschung. Ich fordere Sie eindringlich auf, am 25. September mit JA zu stimmen.

Dr. med. Yves Guisan, Vizepräsident der FMH, Nationalrat 